\title{
Trombosis venosa cerebral como presentación de lupus eritematoso sistémico
}

\author{
P. Cardona-Portela, C. Casasnovas-Pons, M. Moral-Torres, F. Rubio-Borrego
}

\author{
CEREBRAL VENOUS THROMBOSIS AS THE PRESENTING SYMPTOM OF SYSTEMIC LUPUS ERYTHEMATOSUS
}

\begin{abstract}
Summary. Introduction. Cerebral venous thrombosis (CVT) is an infrequent process in systemic lupus erythematosus. We report the case of a female patient whose initial manifestation of lupus was a CVT. Case report. A 30-year-old female who presented headaches and diminished visual acuity; on exploring the patient bilateral papilloedema was found. Magnetic resonance imaging revealed the presence of a venous thrombosis in the superior and transversal longitudinal sinus. Complementary explorations showed high levels of antinuclear antibodies with leukopenia and proteinuria. Antiphospholipid antibodies were negative. Following treatment with anticoagulants, the patient's condition improved both clinically and radiologically. Months later a biopsy was performed and revealed a grade IV diffuse glomerulonephritis. Conclusions. In systemic lupus erythematosus, phenomena such as CVT can be the initial form of presentation of the disease. The presence of antiphospholipid antibodies plays a partial role in CVT; other phenomena, such as inflammatory processes, should also be taken into account. [REV NEUROL 2004; 39: 30-4]
\end{abstract}

Key words. Antiphospholipid. Lupus. Lupus anticoagulant. Systemic lupus erythematosus. Venous thrombosis.

\section{INTRODUCCIÓN}

El lupus eritematoso sistémico (LES) es una enfermedad sistémica con importantes manifestaciones trombóticas arteriales y venosas (en el $10 \%$ de los casos), aunque poco frecuente en el sistema nervioso central (SNC) (en menos del 5\%) [1,2].

La presencia de trombosis venosa cerebral (TVC) en relación con el LES es rara (sólo ocho casos bien documentados), y excepcional como forma de presentación del LES (un caso) [3,4].

La TVC es un proceso vascular en territorio venoso; principalmente, ocurre en los siete senos que constituyen el drenaje principal cerebral. Por extensión o, más raramente, como forma de inicio, acontece en las venas corticales o sistema venoso profundo. Según el tiempo de instauración, se puede clasificar como forma aguda, subaguda o crónica; la segunda es la que encontramos en la mayoría de las ocasiones al realizar el diagnóstico.

La clínica se caracteriza por un síndrome de hipertensión intracraneal determinado por cefalea y papiledema, o bien por una focalidad neurológica, que puede ser negativa, en forma de déficit sensitivos o paresia, o positiva, en forma de crisis parciales o secundariamente generalizadas [5-7].

Raramente, la clínica debuta con un cuadro de encefalopatía subaguda propio del sistema venoso profundo, o hay una clínica más específica, como la que tiene lugar en la trombosis del seno cavernoso, los senos petrosos o la vena yugular, en la que se asocian déficit de pares craneales [8].

$\mathrm{Su}$ diagnóstico se basa en la resonancia magnética (RM) con secuencia de angiorresonancia magnética (ARM), en las que, junto a la localización del trombo, podemos determinar el tiempo de evolución del mismo, según la secuencia de $\mathrm{T}_{2} \mathrm{y}$ el ecogradiente.

La TVC tiene que ser una sospecha diagnóstica en un pa-

Recibido: 18.11.02. Aceptado tras revisión externa sin modificaciones: 27.01.04. Servicio de Neurología. Hospital de Bellvitge. L'Hospitalet de Llobregat, Barcelona, España.

Correspondencia: Dr. Pedro Cardona Portela. Servicio de Neurología. Hospital de Bellvitge. Feixa Llarga, s/n. E-08907 L'Hospitalet de Llobregat (Barcelona).E-mail: 34109pcp@comb.es

(C) 2004, REVISTA DE NEUROLOGÍA ciente joven, preferentemente mujer, con algún factor predisponente a un estado trombofílico. Su diagnóstico no sólo no es importante por cuanto su rápido tratamiento es muy efectivo, sino porque este diagnóstico obliga a descartar una serie de enfermedades y alteraciones de la coagulación, que podrían ser perpetuantes de este estado protrombótico y tratables a la vez que la TVC.

Presentamos una paciente sin enfermedad de base conocida, que muestra un cuadro de cefalea y disminución de la agudeza visual, diagnosticado por RM de TVC. Posteriormente, se hallan datos inmunológicos demostrativos de LES.

\section{CASO CLÍNICO}

Se trata de una mujer de 30 años sin antecedentes patológicos de interés. Durante su estancia en Perú consultó por cefalea hemicraneal derecha pulsátil y vómitos de 24 horas de evolución, sin fiebre ni signos meníngeos, con exploración neurológica normal, excepto por acompañarse de disminución de la agudeza visual y fondo de ojo con la presencia de papiledema bilateral. La RM demostró la presencia de trombosis del seno longitudinal superior y del seno transverso izquierdo (Fig. 1). Se inició un tratamiento con anticoagulantes y corticoides, con el que mejoró inicialmente.

Un mes después acudió a nuestro centro por reaparición de la cefalea con las mismas características que en el episodio previo y parestesias en el hemicuerpo izquierdo.

En la exploración general destacaba livedo reticularis en las extremidades inferiores, con auscultación cardiológica y respiratoria normal. Mostraba un abdomen blando y depresible sin masas ni adenopatías y no refería dolor en la exploración de las articulaciones.

En la exploración neurológica no se hallaron signos meníngeos, las pupilas eran isocóricas y normorreactivas y había disminución de la agudeza visual. La oftalmoscopia reveló papiledema bilateral con componente de microinfarto.

Los pares craneales eran normales; no se apreciaron déficit motores ni sensitivos, ni alteraciones en la coordinación ni en la marcha. Los reflejos miotáticos estaban presentes y eran simétricos. Presentaba RCP flexor bilateral.

En la analítica en sangre destacan: colesterol: 7,08 mmol/L; ALT: 0,63 $\mu \mathrm{kat} / \mathrm{L}$; GGT: 0,72 $\mu \mathrm{kat} / \mathrm{L}$; albúmina: $29 \mathrm{~g} / \mathrm{L}$ (40-50); proteínas: $51 \mathrm{~g} / \mathrm{L}$ (64-80); VSG: $42 \mathrm{~mm} / \mathrm{h}$; plaquetas: $83 \times 10^{9} / \mathrm{mm}^{3}$; leucocitos: $3.150 / \mathrm{L}$ (linfocitos 13\%, neutrófilos 73\%); TTPA (ratio tiempo tromboplastina): 0,97; INR: 1,5; fibrinógeno: 4,94 g/L; hipocomplementemia C3: 47,8 mg/dL (75-140), C3PA: 15 mg/dL (17-25), C4: 16,6 mg/dL (10-40), CH50: $20 \mathrm{U} / \mathrm{mL}$ (51-136); ANA: título 1/320; anti-dADN: $8 \mathrm{U} / \mathrm{mL}(0-6,5)$; antiRO: positivo fuerte; anti-La: positivo débil; antihistona: positivo; anti-Sm: 


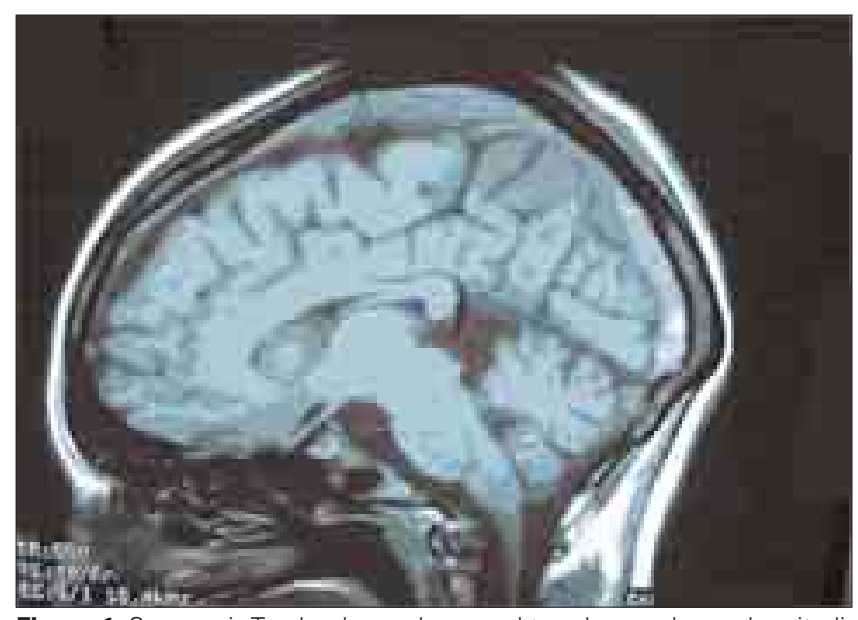

Figura 1. Secuencia $T_{1}$, donde se observa el trombo en el seno longitudinal superior.

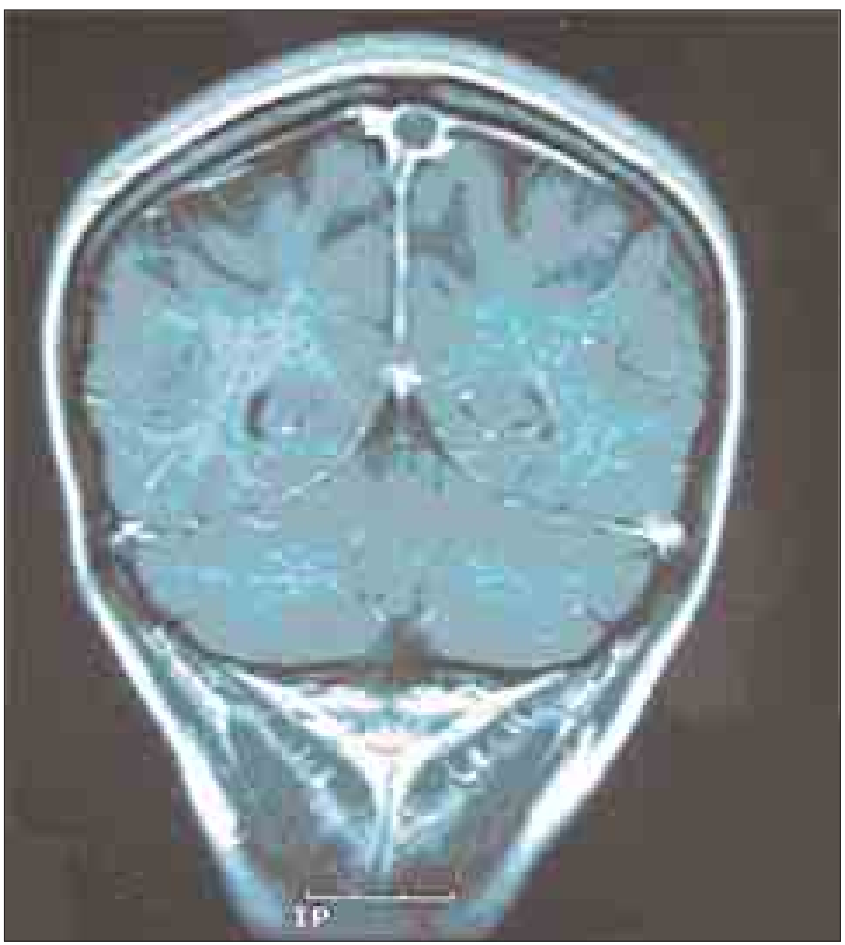

Figura 3. Angiorresonancia magnética: falta de visualización parcial del seno longitudinal superior.

negativo; anticuerpos antifosfolípidos (AAF) y anti- $\mathrm{B}_{2}$ glicoproteína: negativos; c-ANCA y p-ANCA: negativos. Crioglobulinas: positivas. Serologías VIH, CMV, VHC y VHB: negativas. VDRL: negativo. Lyme y Brucella: negativo. Proteinuria de 24 horas: 7,7 g. La radiografía de tórax y el ECG eran normales. La inmunofluorescencia directa de la biopsia de piel fue negativa.

En la TAC craneal se observa dilatación de espacios subaracnoideos de la convexidad cerebral. Después de la administración de contraste, se aprecian signos de trombosis del seno longitudinal.

En la RM se objetiva la recanalización parcial del seno longitudinal superior con persistencia de trombosis del seno transverso izquierdo (Figs. 2 y 3 ).

La ecografía Doppler transcraneal refleja un aumento de velocidad en el sistema venosos profundo.

La paciente, tras el inicio de anticoagulación, presentó una mejoría progresiva del déficit visual, con una RM en la que se objetivaba la recanalización parcial del seno longitudinal superior con persistencia de trombosis del seno tranverso izquierdo (Fig. 4). A los 20 días del ingreso, se apreció la desaparición del papiledema. Los controles analíticos mostraron un consu-

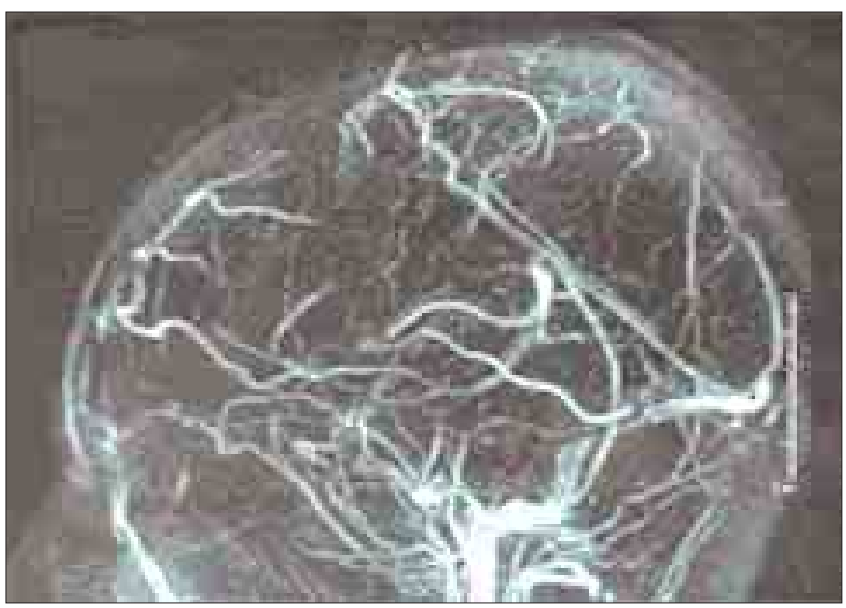

Figura 2. Secuencia $T_{1}$ con gadolinio: trombo en el seno longitudinal superior.

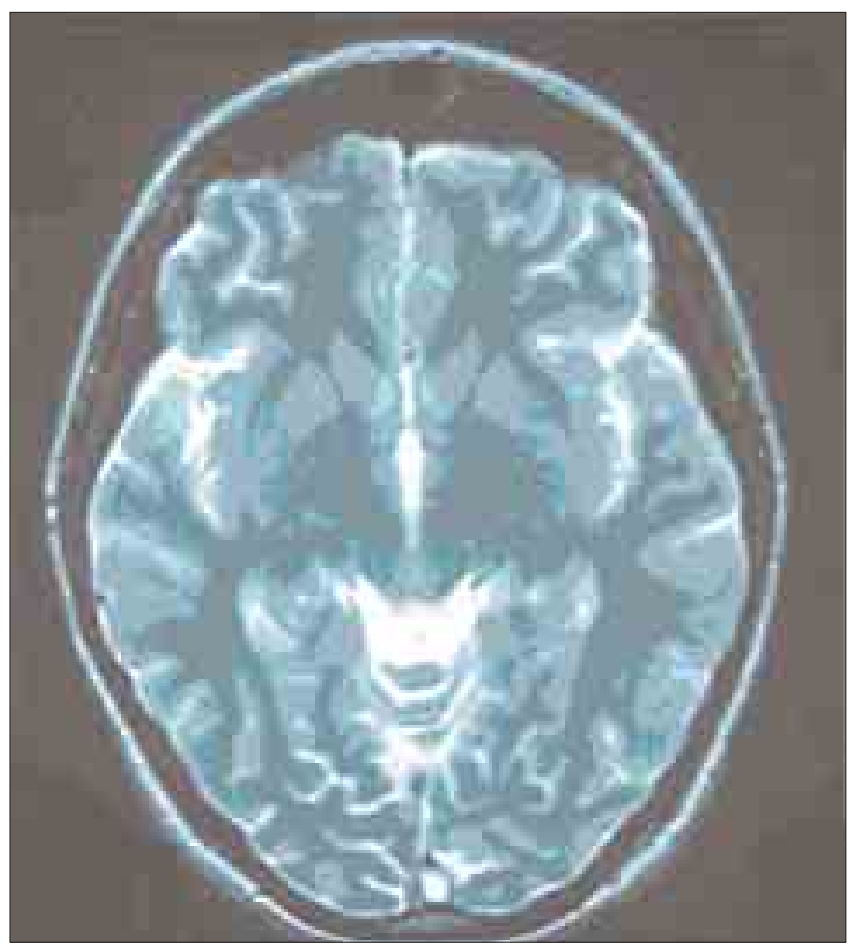

Figura 4. $R M$, secuencia $T_{2}$, que muestra un trombo en el seno longitudinal posterior (20 días después del inicio de la clínica).

mo del complemento, en especial de la fracción CH50, que presentaba un valor de $5 \mathrm{U} / \mathrm{mL}$, ANA con título $1 / 320$ y anti-nADN de $63 \mathrm{U}$, así como un aumento leve de las plaquetas, pero persistía la leucopenia con linfopenia. Destacaba la persistencia de una proteinuria superior a $7 \mathrm{~g} / \mathrm{día}$, con importante presencia de leucocituria y hematuria; por ello, se inició un tratamiento con corticoides en dosis de $60 \mathrm{mg}$ de prednisona al día y se realizó una biopsia renal, que mostró una glomerulonefritis difusa de grado 4 .

Un año después, la paciente se encuentra asintomática, con unos ANA título 1/160, contenido de anti-nADN de $5 \mathrm{U}$, valores de complemento normales, con excepción de CH50, que es de $27 \mathrm{U} / \mathrm{mL}$; la cifra de plaquetas es normal, pero persiste la linfopenia. La RM no muestra nuevos cambios (Fig. 5).

\section{DISCUSIÓN}

Las causas de TVC son muy diversas (Tabla). En el 70\% de los casos son estados protrombóticos primarios o, más frecuentemente, secundarios [2]. 
Entre los primarios se encuentran los déficit de proteína $\mathrm{C}$ y $\mathrm{S}$ -principalmente relacionado con el uso de anticonceptivos orales-; el déficit de antitrombina III, que se transmite con herencia $\mathrm{AD}$-con importantes problemas en el inicio del tratamiento con anticoagulación-; el defecto familiar del plasminógeno, homocistinuria (herencia AR) y la presencia de factor V de Leyden [9].

Este último ha cobrado gran importancia en los últimos años, por su fuerte asociación a fenómenos protrombóticos, principalmente venosos. Consiste en una mutación en el gen que codifica el factor $\mathrm{V}$ y asocia una resistencia a la acción de la proteína C. Su frecuencia en la población es de un 4\%; sin embargo, su asociación a TVC no se excluye, aunque en todos los casos se encuentra otro factor de riesgo, como ha sido el consumo de anticonceptivos orales, la presencia de antifosfolípidos, el síndrome nefrótico o la presencia de alguna enfermedad sistémica, como pudiera ser el LES [10].

Actualmente se están publicando otras alteraciones de la coagulación.

En nuestro caso, si bien la paciente no presentaba ningún signo clínico que orientase a una etiología concreta, la determinación de anticuerpos antinucleares e hipocomplementemia, leucopenia y linfopenia, la anamnesis que demostró una historia de fotosensibilidad, así como la realización de una biopsia renal, constató el diagnóstico de LES.

En el LES existe una serie de alteraciones en la coagulación, y posiblemente en los vasos, que favorece el desarrollo de estados trombofílicos, tanto arteriales como venosos, si bien en la mayoría de los casos acontecen fuera del sistema nervioso.

Dentro de las alteraciones de la coagulación se incluye, por una parte, la pérdida de factores de coagulación e inhibidores de la coagulación por la nefropatía que acompaña al LES. El síndrome nefrótico comporta la eliminación de la antitrombina III, las proteínas $\mathrm{C}$ y S y la acumulación del fibrinógeno.

Por otra parte, contribuye al estado de trombofilia la presencia en el suero de AAF: anticuerpo lúpico (AL) y anticuerpo anticardiolipina (ACL). Su presencia, junto a una clínica de abortos y trombocitopenia, hace sospechar un síndrome antifosfolípido, que se caracteriza, entre otras manifestaciones, por lesiones trombóticas arteriales o venosas e infartos placentarios, y, como consecuencia, abortos.

El síndrome antifosfolípido secundario a lupus tiene un curso clínico independiente de la gravedad y la actividad del lupus.

El anticoagulante lúpico, descrito inicialmente con relación al LES, es un anticuerpo adquirido ( $\operatorname{IgM}, \operatorname{IgG}$ ) que interfiere con los fosfolípidos con carga negativa que intervienen en la cascada de la coagulación y prolonga el TTPA. No se conoce con certeza la fisiopatología de la trombosis con relación a estos anticuerpos. De hecho, se desconoce si son la causa, el resultado, o si son un epifenómeno de la misma. Se supone que la acción de los anticuerpos sobre los fosfolípidos de las membranas endoteliales o de las plaquetas podría provocar la destrucción de éstos y la liberación de diferentes sustancias, como factores plaquetarios y derivados araquidónicos que activarían la coagulación, y ésta actuaría incluso en regiones distantes del lugar de activación.

La presencia de ACL en casos de TVC, sin otra enfermedad autoinmune, oscila entre el 9 y el 22,7\%, según la serie; la presencia de éstos no varia el curso clínico de la trombosis venosa [11].

La presencia de estos anticuerpos, tanto de forma aislada $(3,2 \%)$ o en asociación al LES (20-35\%), se ha relacionado con episodios trombóticos sistémicos y cerebrales, tanto arteriales
Tabla. Causas de trombosis venosa cerebral

Estados protrombóticos

$$
\begin{aligned}
& \text { Puerperio } \\
& \text { Embarazo }
\end{aligned}
$$

Trombofilia hereditaria

Déficit de proteína $\mathrm{S}$

Déficit de proteína C

Déficit de antitrombina III

Resistencia a la proteína $\mathrm{C}$ activa

Mutación del gen de la protrombina

Mutación del factor $\mathrm{V}$ de Leiden

Homoscitinuria

Trombofilia secundaria

Neoplasias

Anemia ferropénica

Policitemia vera

Enfermedades inflamatorias intestinales

Vasculitis

Lupus eritematoso sistémico

Enfermedad de Behçet

Síndrome nefrótico

Síndrome antifosfolípido

Trombosis secundaria a infección

Otitis

Sinusitis

Meningitis

Virus de inmunodeficiencia humana

Empiema subdural

Trombofilia yatrógena

Anticonceptivos orales

Corticosteroides

Catéteres yugulares

Andrógenos

Lasparaginasa

Trombosis secundaria a alteraciones del flujo

Deshidratación

Insuficiencia cardíaca

Fístula arteriovenosa

Tumores locales

Trombosis secundaria a infiltración

Meningitis carcinomatosa

Granulomatosis de Wegener

Sarcoidosis

Miscelánea

Traumatismo craneal

Trombosis venosa asociada

Quiste aracnoideo

Colesteatoma

Enfermedad de von Willebrand

Infección abdominal

Cirugía

Desconocida (30\%) 

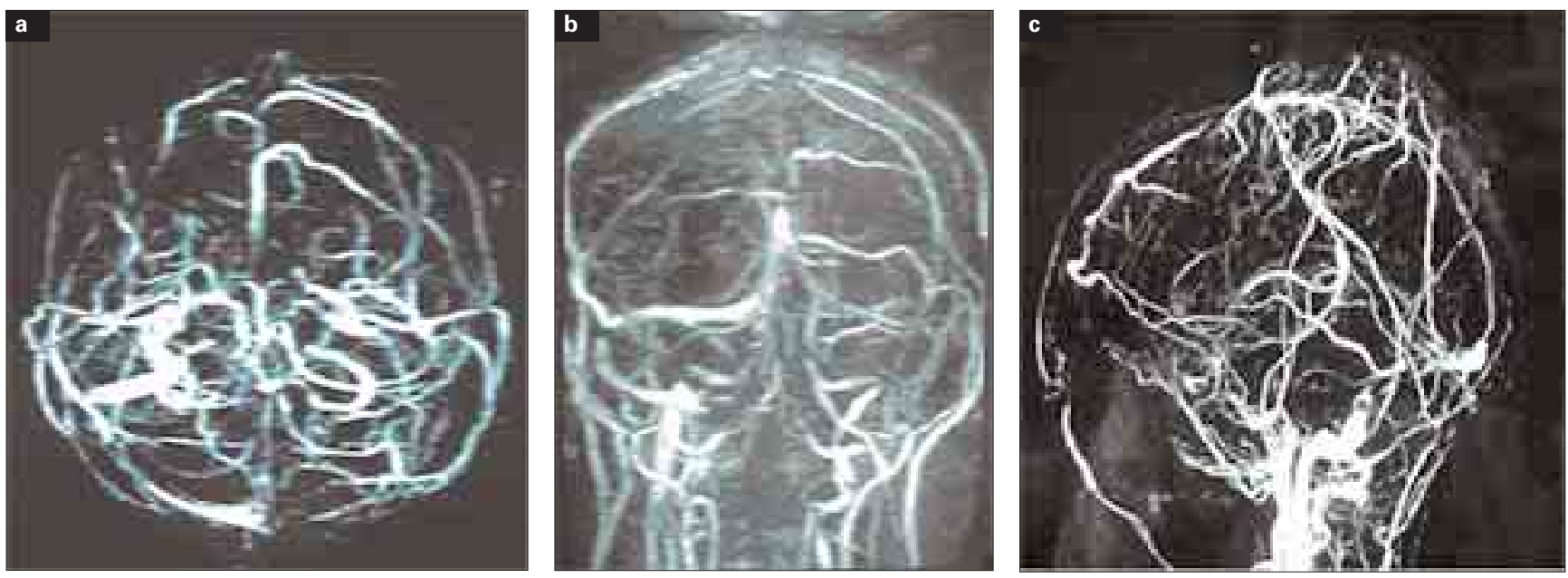

Figura 5. Secuencias de angiorresonancia magnética. Después de un año, muestran todavía el relleno parcial del seno longitudinal superior y la ausencia de flujo venoso en el seno transverso izquierdo.

como venosos. Igualmente, se asocia su presencia en pacientes con LES a trombocitopenia, anemia hemolítica, livedo reticular, enfermedad valvular cardíaca corea y con clínica de cefalea de características migrañosas [12].

La presencia de AAF aumenta de forma considerable el riesgo de eventos trombóticos venosos en paciente con lupus $-50 \%$ de los pacientes con AL positivo frente al $15 \%$ de los que cursan con AL negativo-. Además, su presencia se relaciona con una presentación temprana de dichos eventos.

Los AAF requieren la presencia de una proteína plasmática, la $\mathrm{B}_{2}$-glicoproteína I, para poder unirse a los fosfolípidos; el bloqueo que se produce a esta proteína a través de unos anticuerpos (IgM e IgG glicoproteína) actuaría como factor protrombótico. Si bien su presencia aumenta la mortalidad y los episodios trombóticos arteriales y venosos, se ha demostrado como un factor independiente sobre otras complicaciones sistémicas del LES (nefritis o alteraciones neuropsiquiátricas).

En nuestro caso no se hallaron estos AAF y en series bibliográficas de TVC se detectaron en menos del $30 \%$.

Se cree que, ya sea por los AAF descritos o por otros no identificados hasta el momento, se produce un fenómeno de venulitis que podría ser responsable de la trombosis venosa. Esto se ha observado en el endotelio de paciente con lupus y trombosis, en las extremidades o venosa portal [13].

\section{Tratamiento}

Con independencia de la base etiopatogénica, el tratamiento combinado de anticoagulantes, corticoides y algunos inmunosupresores parece ser el más efectivo en el control de la enfermedad.

El tratamiento anticoagulante es el más eficaz, al igual que en el resto de las TVC, pero su uso combinado con esteroides ha demostrado los mejores resultados en el LES.

El tratamiento anticoagulante parece recomendado principalmente en aquellos LES que presenten, a su vez, AAF, ya sea AL o ACL, como profilaxis primaria. La presencia de éstos de forma aislada sin llegar a cumplir los criterios de LES, no justifica el inicio de la anticoagulación, y en estos casos se optaría por la antiagregación.

El tratamiento a largo plazo con anticoagulación no está bien establecido, aunque se propone un tratamiento indefinido si hay una etiología subyacente que predisponga a la trombosis. El tratamiento con corticoides controlaría, en parte, la respuesta autoinmune y podría conseguir en los casos de síndrome antifosfolípido secundario bajar el tiempo de tromboplastina parcial activada, como parámetro de control.

Otros parámetros de control en relación con el LES podrían ser la concentración de proteínas del complemento, en especial el CH50, de anti-dADA y la VSG, así como una respuesta a la linfopenia. Otros inmunosupresores, como la ciclofosfamida o la azatioprina pueden usarse, de forma única o en asociación con prednisona, como politerapia en el LES, o incluso plantear esplenectomía en los casos que asocian un síndrome antifosfolípido. En casos de cuadros agudos de fallo renal o importante clínica sistémica, se ha llegado a realizar plasmaféresis.

Se ha descrito que, a pesar del tratamiento con corticoides, puede continuar presente el anticoagulante lúpico y persistir los episodios de isquemia cerebral arterial recurrente, incluso con un tratamiento anticoagulante y antiagregante.

En conclusión, ante un nuevo episodio de TVC, debería realizarse una búsqueda de las múltiples enfermedades que podrían asociarse a ésta. A su vez, deberían estudiarse diferentes alteraciones de la coagulación, algunas de ellas congénitas y otras adquiridas, que podrían actuar como factores independientes o asociarse a alguna enfermedad, y que mantendrían y perpetuarían el estado protrombótico dentro del sistema venoso.

Como en nuestra paciente, deberían buscarse alteraciones en diferentes órganos, que inicialmente podrían no orientar a una etiología concreta, pero que en un seguimiento posterior podrían dirigir hacia una causa de la TVC.

En todo paciente con LES deberían determinarse los AAF y anticuerpos como la $\mathrm{B}_{2}$-glicoproteína, ya que se han relacionado con un aumento de problemas trombóticos y son clave para plantear un tratamiento a largo plazo, por el riesgo de recurrencia de la trombosis.

El tratamiento anticoagulante, junto con los corticosteroides, se han mostrado como los más efectivos en el cuadro de trombosis venosa asociada al LES.

Hay que tener en cuenta que, a pesar de una búsqueda exhaustiva, el 30\% de las TVC son de etiología desconocida. Se están describiendo nuevas alteraciones en la coagulación, como 
la mutación 20201A, o la mutación C677T de la MTHFR. Si bien éstas, al igual que los déficit congénitos anteriormente citados, no constituyen por sí mismas factores de riesgo indepen- dientes para la trombosis, su asociación con la toma de anticonceptivos o la presencia de embarazo o alguna enfermedad comporta un factor de riesgo asociado.

\section{BIBLIOGRAFÍA}

1. Brown M, Swash M. Systemic lupus erythematosus. In Vinken PJ, Bruyn GW, Klawans HL, eds. Vascular diseases: handbook of clinical neurology. Vol. 11. Amsterdam: Elsevier; 1989. p. 369-82.

2. Bousser MG, Rossell RR. Cerebral venous thrombosis. Philadelphia: WB Saunders; 1997

3. Kapplan RE, Springate JE, Feld LG, Cohen ME. Pseudotumor cerebri associated with cerebral venous sinus thrombosis, internal jugular vein thrombosis, and systemic lupus erythematosus. J Pediatrics 1985; 107: 266-8.

4. Parnass SM, Goodwin JA. Dural sinus thrombosis: a mechanism for pseudotumor cerebri in systemic lupus erythematosus. J Rheumatol 1987; 14: 152-5.

5. Flusser D, Abu-Shakra M, Baumgarten-Kleiner A, Flusser G, Sukenik S. Superior sagittal sinus thrombosis in a patient with systemic lupus erythematosus. Lupus 1996; 5: 334-6.

6. Vidailhet M, Piette JC. Cerebral venous thrombosis in system lupus erythematosus. Stroke 1990; 21: 1226-31.

\section{TROMBOSIS VENOSA CEREBRAL COMO \\ PRESENTACIÓN DE LUPUS ERITEMATOSO SISTÉMICO}

Resumen. Introducción. La trombosis venosa cerebral (TVC) no es un proceso habitual en el lupus eritematosos sistémico. Presentamos una paciente cuya manifestación inicial del lupus fue una TVC. Caso clínico. Paciente de 30 años de edad que presenta cefalea y disminución de la agudeza visual; en la exploración destaca un papiledema bilateral. La resonancia magnética realizada demuestra la presencia de una trombosis venosa en el seno longitudinal superior y transverso. Las exploraciones complementarias mostraron altas concentraciones de anticuerpos antinucleares con leucopenia y proteinuria. Los anticuerpos antifosfolípidos fueron negativos. Tras un tratamiento anticoagulante, mejoró clínica y radiológicamente. Meses más tarde se realizó una biopsia, que demostró una glomerulonefritis difusa grado IV. Conclusión. En el lupus eritematoso sistémico, fenómenos como la TVC pueden ser la forma de manifestación inicial de la enfermedad. La presencia de anticuerpos antifosfolípidos participa de forma parcial en la TVC; deberían considerarse otros fenómenos, como los inflamatorios. [REV NEUROL 2004; 39: 30-4]

Palabras clave. Anticoagulante lúpico. Antifosfolípido. Lupus. Lupus eritematoso sistémico. Trombosis venosa.
7. Montes de Oca MA, Babron MC. Thrombosis in systemic lupus erythematosus: a French collaborative study. Arch Dis Child 1991; 66: 713-71.

8. Lefkowitz P. Cortical thrombophlebitis and sinovenous disease. In Vinken PJ, Bruyn GW, Klawans HL, eds. Vascular diseases: handbook of clinical neurology. Vol. 10. Amsterdam: Elsevier; 1989. p. 395-423.

9. Hillier CE, Collins PW, Bowen DJ, Bowley S, Wiles CM. Inherited prothrombotic risk factors and cerebral venous thrombosis. Q J Med 1998; 91: 677-80.

10. Deschiens MA, Conard J. Coagulation studies, factor V Leiden, and anticardiolipin antibodies in 40 cases of cerebral venous thrombosis. Stroke 1996; 27: 1724-30.

11. Levin SR, Kieran S. Cerebral venous thrombosis with lupus anticoagulants. Stroke 1987; 18: 801-4.

12. Christopher R, Nagajara D. Anticardiolipin antibodies: a study in cerebral venous thrombosis. Acta Neurol Scand 1999; 99: 121-4.

13. Huizinga TW, Streens SC. Magnetic resonance imaging of large vessel vasculitis. Curr Opin Rheumatol 2001: 13; 41-7.

\section{TROMBOSE VENOSA CEREBRAL COMO APRESENTAÇÃO DE LÚPUS ERITEMATOSO SISTÉMICO}

Resumo. Introdução. A trombose venosa cerebral (TVC) é um processo pouco habitual no lúpus eritematoso sistémico. Apresentamos uma doente cuja manifestação inicial do lúpus foi uma TVC. Caso clínico. Doente de 30 anos de idade que apresenta cefaleia e diminuição da acuidade visual; na exploração destaca-se um papiledema bilateral. A ressonância magnética realizada demonstra a presença de uma trombose venosa no seio longitudinal superior $e$ transverso. As explorações complementares mostraram uns níveis elevados de anti-corpos anti-nucleares com leucopenia e proteinuria. Os anti-corpos antifosfolípidos foram negativos. Após tratamento anti-coagulante, melhorou clínica e radiologicamente. Meses mais tarde realizou-se uma biopsia, que demonstrou uma glomerulonefrite difusa de grau IV. Conclusão. No lúpus eritematoso sistémico, fenómenos como a TVC podem ser a forma de manifestação inicial da doença. A presença de anti-corpos antifosfolipidos participa de forma parcial na TVC; deveriam ser considerados outros fenómenos, como os inflamatórios. [REV NEUROL 2004; 39: 30-4] Palavras chave. Anti-coagulante lúpico. Antifosfolípido. Lúpus. Lúpus eritematoso sistémico. Trombose venosa. 